\title{
DESCRIPCIÓN DE UNA ESPECIE NUEVA DE MILPIÉS DEL GÉNERO BEATADESMUS (DIPLOPODA: POLYDESMIDA: CHELODESMIDAE) PARA LA REPÚBLICA DOMINICANA: NUEVAS SINONIMIAS
}

\author{
Antonio R. Pérez-Asso \\ Investigador Asociado del Museo Nacional de Historia Natural. Plaza de la Cultura, Santo Domingo, República \\ Dominicana. P. O. Box 800846, Coto Laurel PR 00780-0846, Puerto Rico \\ hparaiso@caribe.net
}

\section{RESUMEN}

Se describe una especie nueva de Beatadesmus Loomis para la República Dominicana. Los géneros Quisquicia Loomis y Dendrodesmus Pérez-Asso pasan a ser sinónimos del género Beatadesmus. Se presenta una lista actualizada con las doce especies incluidas actualmente en este género.

Palabras clave: Diplopoda, Polydesmida, Chelodesmidae, Beatadesmus, Quisquicia, Pogonodesmus, Aplopododesmus, Dendrodesmus, nueva especie, nuevas sinonimias.

\section{ABSTRACT}

A new species of the genus Beatadesmus Loomis is described from the Dominican Republic. The genera Quisquicia Loomis and Dendrodesmus Pérez-Asso are placed in synonymy with Beatadesmus. A check list of twelve species currently placed in this genus is included.

Key words: Diplopoda, Polydesmida, Chelodesmidae, Beatadesmus, Quisquicia, Pogonodesmus, Aplopododesmus, Dendrodesmus, new species, new synonymy.

\section{INTRODUCCIÓN}

Loomis (1934) describe el género Beatadesmus a partir de fragmentos de animales muertos y de una hembra viva, pero inmadura, recolectados en la isla Beata, al sur de la República Dominicana; en esa ocasión no fue posible ilustrar los gonopodos de los machos de esa especie, nombrada B. utowani, y solo se presentan los detalles del cuerpo.

En 1936, el mismo autor publica varios géneros relacionados con Beatadesmus, basándose en ejemplares recolectados en Haití, entre ellos nombra a Quisquicia con tres especies (Q. scitula, Q. engonata y $Q$. rubens) y acomoda en este género a Polydesmus sallei DeSaussure, 1860 y a Caraibodesmus insignis Chamberlin, 1918.

A continuación, Loomis (1936) describe los géneros Pogonodesmus (P. pullus) y Aplopododesmus (A. longipes), los cuales exhiben gonopodos semejantes a Quisquicia, pero estructuras corporales diferentes. Acertadamente, Attems (1938) sinonimiza Pogonodesmus y Aplopododesmus y sus especies son ubicadas en el género Quisquicia.

En noviembre de 2005, Pérez-Asso visita la isla Beata y colecta abundante material de Beatadesmus utowani. El objetivo de este viaje era ilustrar los gonopodos de los machos y establecer las posibles relaciones entre Beatadesmus y los otros géneros de chelodésmidos de La Hispaniola. Dicho autor (2008) redescribe entonces $B$. utowani y adicionalmente presenta dos especies nuevas (B. ivonneae y $B$. pedernales) para localidades cercanas a la isla Beata en la península de Barahona.

En el presente trabajo se establece que el género Quisquicia puede ser acomodado como sinónimo de Beatadesmus. Ya Loomis (1936) comenta en la descripción de Quisquicia que muchos caracteres estructurales la asocian con Beatadesmus y que el examen de los gonopodos de Beatadesmus podría definir esta situación. También Hoffman (1999) plantea la probabilidad de considerar a Quisquicia un sinónimo de Beatadesmus. Además, se establece que el género Dendrodesmus (D. yuma) publicado por Pérez-Asso (2003) tampoco se sustenta, por lo que debe ser considerado también como un sinónimo de Beatadesmus. Adicionalmente se presenta una especie nueva, lo que aumenta a doce las especies del género Beatadesmus en la isla de La Hispaniola. 


\title{
MATERIALES Y MÉTODOS
}

Los especímenes recolectados fueron conservados cuidadosamente, quedando perfectamente aplanados, lo que facilita notablemente su estudio. La longitud total se determinó midiendo los ejemplares desde la cabeza hasta el ápice del epiprocto, el ancho se tomó al nivel del séptimo segmento entre los ángulos posteriores de los paraterguitos. Las ilustraciones se realizaron con cámara lúcida en un microscopio Wild MSD. En las láminas donde aparecen ilustrados los gonopodos, el proceso prefemoral se oscureció para facilitar su observación y resaltar el contraste con el acropodito. Los ejemplares se encuentran depositados en la colección ARPA(colección personal del autor).

\section{RESULTADOS}

\author{
SISTEMÁTICA \\ Género Beatadesmus Loomis
}

Beatadesmus Loomis, 1934, Smithsonian Misc. Coll., 89 (14): 31-32. Especie tipo: B. utowani Loomis. Hoffman, 1999, VMNH Spec. Publ., 8: 284. Pérez-Asso y Pérez-Gelabert, 2001, Bol. SEA, 28: 74. PérezAsso, 2008, Solenodon, 7: 14-28.

Quisquicia Loomis, 1936, Bull. Mus. Comp. Zool., 80: 128. Especie tipo: Q. scitula Loomis. Hoffman, 1999, VMNH Spec. Publ., 8: 290. Pérez-Asso y Pérez-Gelebert, 2001, Bol. SEA, 28: 75. SINÓNIMO NUEVO.

Dendrodesmus Pérez-Asso, 2003, Solenodon, 3: 57. Especie tipo: D. yuma Pérez-Asso. SINÓNIMO NUEVO.

Pogonodesmus Loomis, 1936, Bull. Mus. Comp. Zool., 80: 137. Especie tipo: P. pullus Loomis. Sinonimizado por Attems, 1938 e incluido en Quisquicia (Q. pullus) y corregido por Hoffman, 1999 (Q. pulla).

Aplopododesmus Loomis, 1936, Bull. Mus. Comp. Zool., 80: 139. Especie tipo: A. longipes Loomis. Sinonimizado por Attems, 1938 e incluido en Quisquicia (Q. longipes).

Comentario. Aunque las formas y estructuras del cuerpo de las especies pertenecientes a los géneros Beatadesmus, Quisquicia y Dendrodesmus son extremadamente variables -ya que pueden presentar marcada diferencia de color entre los segmentos poríferos y no poríferos, o por el contrario, todos los segmentos similares en colorido; el dorso liso, con gránulos o hileras de macrosetas; el margen posterior de los paraterguitos liso o con varios procesos dentiformes; los poros abriendo lateral o dorsalmente desde el margen ligeramente engrosado del paraterguito o desde prominentes callos poríferos- por solo mencionar algunos de los caracteres más sobresalientes; todas estas especies, ahora acomodadas en el prioritario género Beatadesmus, poseen en común una gran similitud en el patrón estructural de los gonopodos, los cuales son simples, con el acropodito alargado y curvado hacia dentro y el proceso prefemoral básicamente también alargado y curvado hacia dentro (inusualmente aplanado o lobulado). Esta variabilidad en la morfología del cuerpo y la similitud en las estructuras gonopodales ya la hemos encontrado en otros géneros de chelodésmidos antillanos, como por ejemplo en Amphelictogon (PérezAsso, 1996, 1998) con 21 especies confirmadas y en Achromoporus (Pérez-Asso, 2009) con 17 especies conocidas.

\section{Género Beatadesmus Loomis}

Diagnosis. Tamaño variable (16- $40 \mathrm{~mm}$ ). Dorso liso, granuloso, o con hileras de macrosetas. Segmentos poríferos y no poríferos similares o de diferente color. Paraterguitos de la porción anterior del cuerpo con diente en el ángulo anterior. Ángulo posterior de los paraterguitos no poríferos usualmente agudo y prominente. Margen posterior de los paraterguitos usualmente con uno o más procesos dentiformes, inusualmente liso. Fórmula porífera normal ( 5, 7, 9-10, 12-13, 15-19). Los poros abren lateralmente desde un callo porífero prominente, o abren lateral o dorsalmente desde el margen ligeramente engrosado. Gonopodos simples, el acropodito alargado, ligeramente curvado hacia dentro, usualmente con lóbulo redondeado o agudo en la zona de contacto con el postfémur, el cual es filiforme; proceso prefemoral también alargado y curvado hacia dentro, inusualmente aplanado, o lobulado. 
Diagnosis. Variable size, from $16 \mathrm{~mm}$ to $40 \mathrm{~mm}$. Dorsum smooth, with nodules, or row of macrosetae. Poriferous and non-poriferous segments with similar or different color. Scapuloral dentation present at the anterior portion of the body. Posterior corner of non-poriferous paranota sharp and prominent. Posterior margin of paranota usually with one or more teeth, or smooth. Pore formula normal $(5,7,9-10,12-13,15-$ 19). Ozopore opening laterally from a well defined peritremata, or ozopore opening laterally or dorsally from a slightly thickened margin. Simple gonopods, with elongated acropodite, slightly curving inwards, usually with rounded or sharp lobe in its terminal portion; postfemur simple, filiform; prefemoral process also elongated and curving inwards, sometimes flattened or lobed.

\section{Beatadesmus bahorucoensis sp. nov.}

(Figs. 1-2)

Diagnosis. Tamaño mediano (hasta $34 \mathrm{~mm}$ ). No muestra diferencia de color entre segmentos poríferos y no poríferos (Fig. 1A, C). Usualmente presenta diente diminuto en el ángulo anterior de los paraterguitos de los segmentos 2-9 (Fig. 1B). Dorso con una hilera transversal de macrosetas sobre cada segmento (Fig. 1A-C). El poro abre lateralmente desde un prominente callo porífero (Fig. 1A-C). Hypoprocto triángular y ligeramente hinchado hacia la porción apical (Fig. 1D). Gonopodos notablemente simples; acropodito alargado, curvado hacia dentro en su porción terminal, agudo en el ápice (Fig. 2A-D); proceso prefemoral también alargado, más ancho en la porción media, filiforme y curvado hacia dentro en su porción terminal (Fig. 2A-C, E).

Diagnosis. Medium size (up to $34 \mathrm{~mm}$ ). Poriferous and non-poriferous segments with similar color pattern (Fig. 1A, C). Scapuloral dentation usually in segments 2-9 (Fig. 1B). Dorsum with a transverse row of macrosetae on each segment (Fig. 1A-C). Ozopore opening laterally from a well defined peritremata (Fig. 1A-C). Hypoproct triangular and slightly swollen apically (Fig. 1D). Gonopods very simple; acropodite curving inwards, acute at apex (Fig. 2A-D); prefemoral process also elongated, wide in its central portion, filiform and curving inwards apically (Fig. 2A-C, E).

Holotipo. Macho 5884, largo $28.5 \mathrm{~mm}$, ancho $3.8 \mathrm{~mm}$. Cuerpo aplanado dorso-ventralmente, liso. Collum con macrosetas aisladas; pareterguitos y metazonitos de todo el cuerpo con una hilera de macrosetas situadas básicamente hacia la región anterior de cada segmento, esta línea de macrosetas no excede usualmente el número de 9 por segmento, además presentan macrosetas aisladas y no alineadas, segmento 19 con la hilera de macrosetas situadas cerca del margen posterior. Cabeza, antenas y collum castaño rojizo; segmentos con prozonitos, pareterguitos, pleuritos y esternitos castaño rojizo, solo los metazonitos muestran la mitad anterior castaño rojizo y la mitad posterior castaño claro; patas castaño claro amarillento; epiprocto castaño rojizo, hypoprocto y valvas anales castaño claro. Ángulo anterior de los paraterguitos 2-9 redondeado y con diente diminuto, margen lateral liso, ángulo posterior agudo y prominente hacia la región media y posterior del cuerpo, margen posterior de los paraterguitos de los segmentos de la región media y posterior del cuerpo con un pequeño diente. Coxas del segundo par de patas portando un tubérculo redondeado.

Variabilidad. Machos: largo 28-30 mm, ancho 3.5-3.8 mm. Hembras: largo 31-34 mm, ancho 3.8-4.0 mm. Espécimen macho 5885 con dos dientes en el margen posterior del paraterguito de los segmentos 5-11, el diente interno diminuto, semejante a un nódulo redondeado, el externo ligeramente mayor. Hembra 5888 con diente en el ángulo anterior de los paraterguitos de los segmentos 2-7. El poro puede abrir lateralmente $\mathrm{o}$, a veces, algo dorsalmente desde un prominente callo porífero.

Tipos. Holotipo, macho 5884. Paratipos, machos 5885-5887, hembra 5888, macho juvenil 5889, hembras juveniles 5890-5892; HISPANIOLA, República Dominicana, Las Abejas (1,200 msnm), Sierra de Bahoruco; en humus y bajo troncos podridos, en bosque latifoliado muy húmedo, rodeado de pinares; A. R. Pérez-Asso; 8-XI-2005. Machos 7960-7962, hembras 7963-7966, macho juvenil 7967; Las Abejas, Sierra de Bahoruco; en humus y hojarasca; A. R. Pérez-Asso; 23-XI-2008.

Material adicional. Machos 7661-7681, hembras 7682-7694, juveniles 7695-7699; HISPANIOLA, República Dominicana, Caseta 2 (1,700 msnm), Sierra de Bahoruco; en humus y hojarasca, en bosque latifoliado rodeado de pinares; A. R. Pérez-Asso y H. Andujar; 22-XI-2008.

Nota. Los especímenes 7665 (macho) y 7693 (hembra) fueron depositados en la colección de milpiés del Museo Nacional de Historia Natural de Santo Domingo, República Dominicana. 

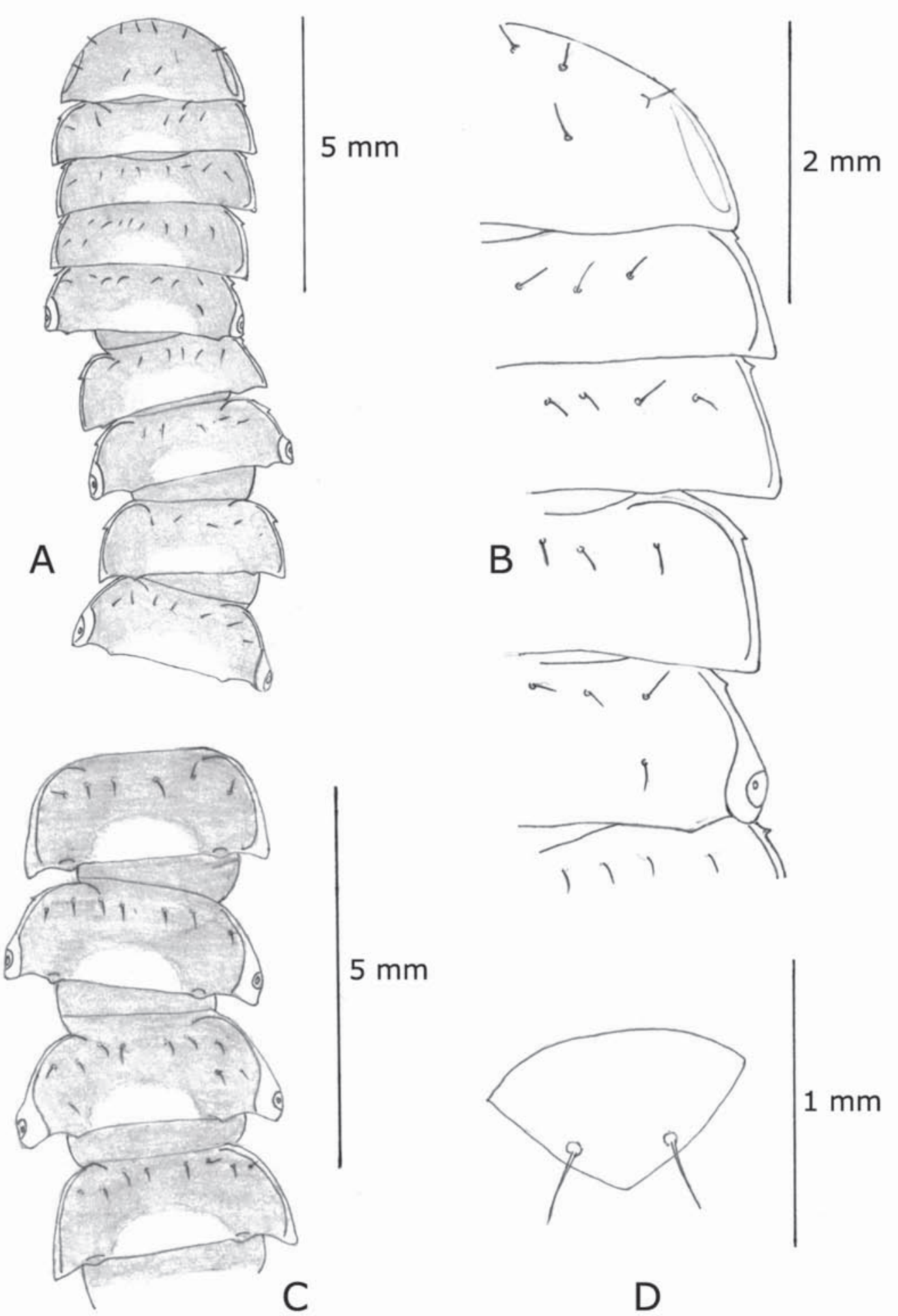

Figura 1. Cuerpo de Beatadesmus bahorucoensis sp. nov. A-B. Aspecto dorsal de los segmentos anteriores del cuerpo. C. Segmentos 8-11. D. Hypoprocto. 

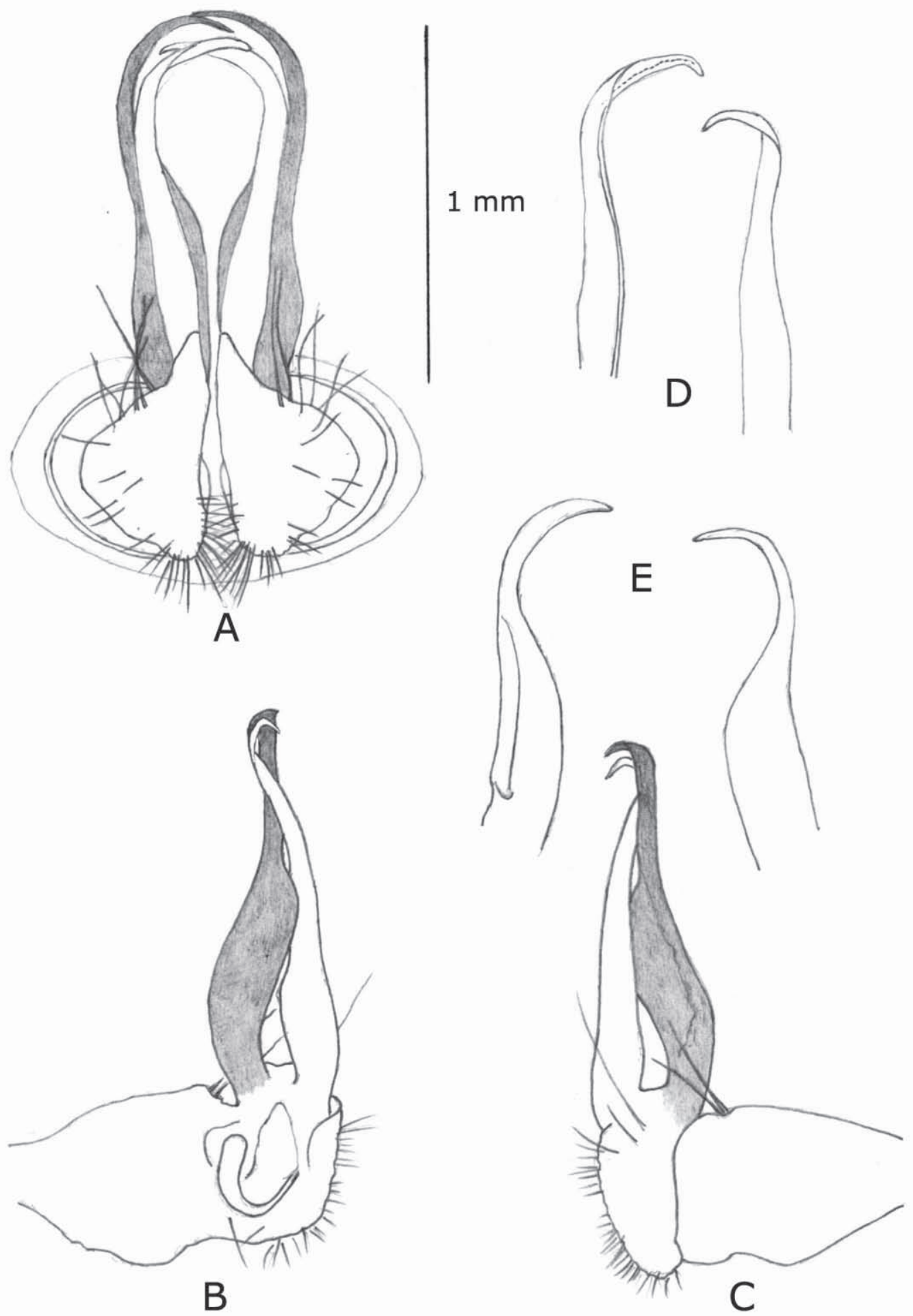

Figura 2. Gonopodos de Beatadesmus bahorucoensis sp. nov. A. Vista ventral. B. Gonopodo izquierdo (vista anterior). C. Gonopodo izquierdo (vista lateral). D. Acropodito (diferentes vistas). E. Proceso prefemoral (diferentes vistas). 
Etimología. El epíteto específico alude a la Sierra de Bahoruco, localidad donde habita esta especie nueva.

Distribución geográfica. Conocida solo de la localidad tipo.

\section{CLAVE TAXONÓMICA PARA LOS GÉNEROS DE CHELODESMIDAE DE LA HISPANIOLA*}

1. Gonopodos pequeños, los cuales no alcanzan el borde anterior del esternito del séptimo segmento ---------------------------- Podiscodesmus

Gonopodos que sobrepasan el borde anterior del esternito del

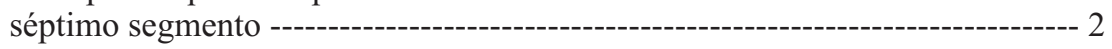

2. Gonopodos con acropodito birramoso --------------------------- Achromoporus Gonopodos con acropodito no birramoso --------------------------------- 3

3. Gonopodos con acropodito plegado sobre si mismo (en zig-zag) en su porción basal (cerca del prefémur) -------------------- Chondrotropis Gonopodos con acropodito no plegado en su porción basal ------------------ 4

4. Gonopodos con acropodito plegado en su porción distal -------- Hypselodesmus Gonopodos con acropodito no plegado en su porción distal ------------------ 5

5. Gonopodos con el proceso prefemoral curvado hacia dentro --- Beatadesmus Gonopodos con el proceso prefemoral curvado hacia fuera ------ Cyrtaphe

* Los géneros Ellipodesmus Chamberlin y Pterigiodesmus Loomis fueron descritos en base a ejemplares hembras; por tanto, no se conocen los gonopodos y no se incluyen en la clave.

Lista actualizada de las especies de Beatadesmus Loomis

Beatadesmus bahorucoensis sp. nov.

Beatadesmus engonatus (Loomis)

Quisquicia engonata Loomis, 1936. Haití.

Beatadesmus insignis (Chamberlin)

Caraibodesmus insignis Chamberlin, 1918. Haití.

Quisquicia insignis: Loomis, 1936.

Beatadesmus ivonneae Pérez-Asso

Beatadesmus ivonneae Pérez-Asso, 2008. República Dominicana.

Beatadesmus longipes (Loomis)

Aplopododesmus longipes Loomis, 1936. Haití.

Quisquicia longipes: Attems, 1938.

Beatadesmus pedernales Pérez-Asso

Beatadesmus pedernales Pérez-Asso, 2008. República Dominicana.

Beatadesmus pullus (Loomis)

Pogonodesmus pullus Loomis, 1936. Haití.

Quisquicia pullus: Attems, 1938.

Quisquicia pulla: Hoffman, 1999.

Beatadesmus rubens (Loomis)

Quisquicia rubens Loomis, 1936. Haití.

Beatadesmus sallei (DeSaussure)

Polydesmus (Leptodesmus) Sallei DeSaussure, 1860. L’ile de Saint-Domingue.

Odontopeltis sallei: Pocock, 1894.

Caraibodesmus (¿) sallei: Chamberlin, 1918.

Quisquicia sallei: Loomis, 1936.

Beatadesmus scitulus (Loomis)

Quisquicia scitula Loomis, 1936. Haití.

Beatadesmus utowani Loomis

Beatadesmus utowani Loomis, 1934. República Dominicana.

Beatadesmus yuma (Pérez-Asso)

Dendrodesmus yuma Pérez-Asso, 2003. República Dominicana. 


\section{AGRADECIMIENTOS}

Mi agradecimiento a Héctor Andújar (Grupo Jaragua) por su valiosa ayuda en las colectas y por su desempeño como chofer y guía en las expediciones. A Yvonne Arias (Grupo Jaragua) por su apoyo con el transporte y por sus recomendaciones durante las visitas a localidades remotas en la República Dominicana. A Cecilia Hernández (Ministerio de Medio Ambiente y Recursos Naturales) por facilitar los permisos de exportación de especímenes, a Matilde Mota y Germán Dominici (Viceministerio de Áreas Protegidas y Biodiversidad de la República Dominicana) por su ayuda en la obtención de los permisos para el trabajo de campo. A Celeste Mir y a Carlos Suriel por la ayuda brindada en el Museo Nacional de Historia Natural, Santo Domingo; Suriel también contribuyó haciendo la revisión del manuscrito. A Víctor González, nuestro patrocinador.

\section{LITERATURA CITADA}

Attems, C. M.T.Graf von. 1938. Fam. Leptodesmidae, Platyrhachidae, Oxydesmidae, Gomphodesmidae, in Das Tierreich, 69: 1-487.

Hoffman, R. L. 1999. Checklist of the millipeds of North and Middle America. Virginia Mus. Nat. Hist., Spec. Publ., 8: 1-584.

Loomis, H. F. 1934. Millipeds of the West Indies and Guiana collected by the Allison V. Armour Expedition in 1932. Smithsonian Misc. Coll., 89 (14): 1-69.

Loomis, H. F. 1936. The millipeds of Hispaniola, with descriptions of a new family, new genera, and new species. Bull. Mus. Comp. Zool., 80: 3-191.

Pérez-Asso, A. R. 1996. Revisión del género Amphelictogon (Diplopoda: Polydesmida: Chelodesmidae) en Cuba. Insecta Mundi 10 (1-4): 181-216.

Pérez-Asso, A. R. 1998. Nuevas especies del género Amphelictogon (Diplopoda: Polydesmida: Chelodesmidae) en Cuba. Insecta Mundi 12 (3-4): 161-173.

Pérez-Asso, A. R. 2003. Nuevo género y especie de milpiés de la familia Chelodesmidae (Diplopoda: Polydesmida) para la Hispaniola. Solenodon 3: 57-60.

Pérez-Asso, A. R. 2008. El género Beatadesmus (Diplopoda: Polydesmida: Chelodesmidae) en la Hispaniola. Solenodon 7: 14-28.

Pérez-Asso, A. R. 2009. El género Achromoporus (Diplopoda: Polydesmida: Chelodesmidae) en la República Dominicana: especies nuevas y sinonimias. Solenodon 8: 33-81.

Pérez-Asso, A. R. y D. E. Pérez-Gelabert. 2001. Checklist of the millipeds (Diplopoda) of Hispaniola. Boletin S.E.A., 28: 67-80. 